PROCEEDINGS OF THE

AMERICAN MATHEMATICAL SOCIETY

Volume 134, Number 9, September 2006, Pages 2541-2548

S 0002-9939(06)08291-8

Article electronically published on March 23, 2006

\title{
REGULARIZED PRODUCT EXPRESSIONS OF HIGHER RIEMANN ZETA FUNCTIONS
}

\author{
TETSUYA MOMOTANI
}

(Communicated by Wen-Ching Winnie Li)

\begin{abstract}
As a generalization of recent work by Kurokawa, Matsuda, and Wakayama (2004) we introduce a higher Riemann zeta function for an abstract sequence. Then we explicitly determine its regularized product expression.
\end{abstract}

\section{IntRoduction}

It is known ( $\overline{\mathrm{De}})$ that the Riemann zeta function $\zeta(s)=\prod_{p \text { :prime }}\left(1-p^{-s}\right)^{-1}$ has the regularized product expression

$$
\prod_{\rho \in R}\left(\frac{s-\rho}{2 \pi}\right)=2^{-1 / 2}(2 \pi)^{-2} \pi^{-s / 2} s(s-1) \Gamma\left(\frac{s}{2}\right) \zeta(s),
$$

where $R$ is the set of the non-trivial zeros of $\zeta(s)$, and $\Gamma(s)$ is the classical gamma function.

On the other hand, a higher Riemann zeta function $\zeta_{l \infty}(s):=\prod_{n=1}^{\infty} \zeta(s+\ln )$ has been introduced and studied in the paper [CL for $l=1$, [KMW] for $l \in \mathbb{Z}_{\geq 1}$. We now consider a generalized higher Riemann zeta function. Let $\Lambda=\left\{\lambda_{k}\right\}_{k \in I}$ be a sequence of complex numbers. Then we define a higher Riemann zeta function for the sequence $\Lambda$ by

$$
Z(s, \Lambda):=\prod_{\lambda \in \Lambda} \prod_{p: \text { prime }}\left(1-p^{-s-\lambda}\right)^{-1}=\prod_{\lambda \in \Lambda} \zeta(s+\lambda) .
$$

In this paper, we study several properties of the higher Riemann zeta function. For a "regularizable" sequence $\Lambda$, we see that $Z(s, \Lambda)$ has the regularized product expression

$$
\bigoplus_{\lambda \in \Lambda, \rho \in R}\left(\frac{s+\lambda-\rho}{2 \pi}\right)=\text { " gamma factor " } \times Z(s, \Lambda) .
$$

Here $\boxminus$ denotes the "dotted" regularized product due to [KW3.

Received by the editors January 11, 2005 and, in revised form, April 10, 2005.

2000 Mathematics Subject Classification. Primary 11M36, 11M06.

Key words and phrases. Riemann's zeta function, multiple gamma functions, regularized products.

(C)2006 American Mathematical Society Reverts to public domain 28 years from publication 
Further, when $\Lambda$ is given by a lattice $\Omega:=\left\{n_{1} \omega_{1}+\cdots+n_{r} \omega_{r} \mid n_{j} \in \mathbb{Z}_{\geq 0}\right\}$, we show that the higher Riemann zeta function

$$
Z(s, \Omega)=\prod_{n_{1}, \cdots, n_{r}=0}^{\infty} \prod_{p: \text { prime }}\left(1-p^{-\left(s+n_{1} \omega_{1}+\cdots+n_{r} \omega_{r}\right)}\right)^{-1}
$$

satisfies a certain functional equation of the type $s \leftrightarrow 1+\omega_{1}+\cdots+\omega_{r}-s$. We remark that the form of $Z(s, \Omega)$ looks like the Selberg zeta function (when $r=1$ ) defined for a discrete subgroup of a real rank 1 semi-simple Lie group (cf. Go Ku2]).

\section{Notation}

2.1. Regularized product. Let $\Lambda=\left\{\lambda_{k}\right\}_{k \in I}$ be a sequence of complex numbers. We call the sequence $\Lambda$ "regularizable" if $\Lambda$ satisfies the following conditions: (i) $\operatorname{Re}\left(\lambda_{k}\right) \geq 0$ for all $k \in I$, and $\operatorname{Re}\left(\lambda_{k}\right) \rightarrow \infty$ as $k \rightarrow \infty$. (ii) The series $\sum_{k \in I, \lambda_{k} \neq 0} \lambda_{k}^{-s}$ converges for sufficiently large $\operatorname{Re}(s)$. (iii) The function $\Theta(x, \Lambda):=\sum_{k \in I} e^{-\lambda_{k} x}$ has an asymptotic expansion

$$
\Theta(x, \Lambda) \sim \sum_{n=0}^{\infty} x^{t_{n}} T_{n}(\log x) \quad \text { as } x \rightarrow+0,
$$

where $t_{0} \leq 0, t_{0}<t_{1}<t_{2}<\cdots \rightarrow+\infty$, and $T_{n}(z) \in \mathbb{C}[z]$ is a polynomial.

For a regularizable sequence, we can define the regularized product as follows.

Lemma 2.1 ([Il], KW3]). Assume that the sequence $\Lambda=\left\{\lambda_{k}\right\}_{k \in I}$ is regularizable. Then, (1) for $\operatorname{Re}(z)>0$, the function

$$
\zeta(s, z, \Lambda):=\sum_{k \in I}\left(z+\lambda_{k}\right)^{-s}
$$

has an analytic continuation as a meromorphic function on $s \in \mathbb{C}$. (2) The "dotted" regularized product

$$
\bigoplus_{k \in I}\left(z+\lambda_{k}\right):=\exp \left\{-\mathrm{CT}_{s=0} \frac{\zeta(s, z, \Lambda)}{s}\right\}
$$

exists, and this is an entire function on $z \in \mathbb{C}$ with zeros at $z=-\lambda_{k}(k \in I)$. Here $\mathrm{CT}_{s=0} f(s)$ denotes the constant term in the Laurent expansion of $f(s)$ at $s=0$.

We remark that the dotted regularized product $\bigoplus_{m \in I, n \in J}\left(z+a_{m}+b_{n}\right)$ exists if $\left\{a_{m}\right\}_{m \in I}$ and $\left\{b_{n}\right\}_{n \in J}$ are regularizable sequences. This follows at once from the relation $\Theta\left(x,\left\{a_{m}+b_{n}\right\}_{m \in I, n \in J}\right)=\Theta\left(x,\left\{a_{m}\right\}_{m \in I}\right) \times \Theta\left(x,\left\{b_{n}\right\}_{n \in J}\right)$.

2.2. Multiple gamma function and multiple sine function. Let $\boldsymbol{\omega}=$ $\left(\omega_{1}, \cdots, \omega_{r}\right)$, where $\omega_{j} \in \mathbb{C}, \operatorname{Re}\left(\omega_{j}\right)>0$, and $\Omega:=\left\{n_{1} \omega_{1}+\cdots+n_{r} \omega_{r} \mid n_{j} \in \mathbb{Z}_{\geq 0}\right\}$. We fix $\operatorname{Re}(z)>0$. Then the multiple zeta function of Barnes $([\mathrm{Ba}])$ is defined by

$$
\zeta(s, z, \boldsymbol{\omega}):=\zeta(s, z, \Omega)=\sum_{n_{1}, \cdots, n_{r}=0}^{\infty}\left(z+n_{1} \omega_{1}+\cdots+n_{r} \omega_{r}\right)^{-s} .
$$

This sum converges absolutely for $\operatorname{Re}(s)>r$. It is seen that the sequence $\Omega$ is regularizable, and $\zeta(s, z, \boldsymbol{\omega})$ has a meromorphic continuation to $s \in \mathbb{C}$. Further, for 
$m \in \mathbb{Z}_{\geq 0}$, it is known that

$$
\zeta(-m, z, \boldsymbol{\omega})=\frac{(-1)^{m} \cdot m ! \cdot B_{m+r}(z, \boldsymbol{\omega})}{(m+r) !} .
$$

Here $B_{n}(z, \boldsymbol{\omega})$ is the multiple Bernoulli polynomials given by

$$
\frac{x^{r} e^{-z x}}{\left(1-e^{-\omega_{1} x}\right) \cdots\left(1-e^{-\omega_{r} x}\right)}=\sum_{n=0}^{\infty} \frac{B_{n}(z, \boldsymbol{\omega})}{n !} x^{n},
$$

for $|x|<\min _{1 \leq j \leq r}\left|2 \pi / \omega_{j}\right|$. We remark that $\zeta(s)=\zeta(s, 1,(1))$ is the Riemann zeta function and $\zeta(-m)=(-1)^{m} B_{m+1} /(m+1)$, where $B_{m}=B_{m}(1,(1))$ is the usual Bernoulli number.

Now we define the multiple gamma function $\Gamma(z, \boldsymbol{\omega})$ and the multiple sine function $S(z, \boldsymbol{\omega})$ by using the regularized product as follows ([Ku1]):

$$
\begin{aligned}
& \Gamma(z, \boldsymbol{\omega}):=\exp \left\{\left.\frac{d}{d s} \zeta(s, z, \boldsymbol{\omega})\right|_{s=0}\right\}=\prod_{n_{1}, \cdots, n_{r}=0}^{\infty}\left(z+n_{1} \omega_{1}+\cdots+n_{r} \omega_{r}\right)^{-1}, \\
& S(z, \boldsymbol{\omega}):=\Gamma(z, \boldsymbol{\omega})^{-1} \cdot \Gamma\left(\omega_{1}+\cdots+\omega_{r}-z, \boldsymbol{\omega}\right)^{(-1)^{r}} .
\end{aligned}
$$

It is seen that these functions satisfy the conditions

$$
\begin{aligned}
& \Gamma\left(z,\left(\omega_{1}, \cdots, \omega_{r-1}, \omega_{r}\right)\right)=\Gamma\left(z,\left(\omega_{1}, \cdots, \omega_{r-1}\right)\right) \cdot \Gamma\left(z+\omega_{r},\left(\omega_{1}, \cdots, \omega_{r-1}, \omega_{r}\right)\right), \\
& S\left(z,\left(\omega_{1}, \cdots, \omega_{r-1}, \omega_{r}\right)\right)=S\left(z,\left(\omega_{1}, \cdots, \omega_{r-1}\right)\right) \cdot S\left(z+\omega_{r},\left(\omega_{1}, \cdots, \omega_{r-1}, \omega_{r}\right)\right) .
\end{aligned}
$$

We also note that $\Gamma(z,(1))=(2 \pi)^{-1 / 2} \Gamma(z)$ and $S(z,(1))=2 \sin (\pi z)$.

\section{Higher Riemann zeta function}

3.1. Definition and analytic continuation. First of all, we introduce a higher Riemann zeta function as follows.

Definition 3.1. Define a higher Riemann zeta function for $\Lambda=\left\{\lambda_{k}\right\}_{k=0}^{\infty}$ by

$$
Z(s, \Lambda)=Z\left(s,\left\{\lambda_{k}\right\}_{k=0}^{\infty}\right):=\prod_{k=0}^{\infty} \zeta\left(s+\lambda_{k}\right)=\prod_{k=0}^{\infty} \prod_{p: \text { prime }}\left(1-p^{-\left(s+\lambda_{k}\right)}\right)^{-1} .
$$

To show the absolute convergence of $Z(s, \Lambda)$, we prepare the following lemma.

Lemma 3.2. Fix a positive number $x \geq 2$. If the sequence $\Lambda=\left\{\lambda_{k}\right\}_{k=0}^{\infty}$ satisfies (i) $0 \leq \operatorname{Re}\left(\lambda_{0}\right) \leq \operatorname{Re}\left(\lambda_{1}\right) \leq \cdots \rightarrow \infty$, and (ii) the sum $\sum_{k \in I, \lambda_{k} \neq 0} \lambda_{k}^{-s}$ converges absolutely for large $\operatorname{Re}(s)$, then we have

$$
\left|\sum_{k=j}^{\infty} x^{-\lambda_{k}}\right| \ll_{j} x^{-\operatorname{Re}\left(\lambda_{j}\right)}, \quad \text { for all } j \geq 0
$$

Proof. We observe that

$$
\begin{aligned}
\left|\sum_{k=j}^{\infty} x^{-\lambda_{k}}\right| & =\left|x^{-\lambda_{j}} \cdot \sum_{k=j}^{\infty} x^{-\left(\lambda_{k}-\lambda_{j}\right)}\right| \\
& \leq x^{-\operatorname{Re}\left(\lambda_{j}\right)} \cdot \sum_{k=j}^{\infty} 2^{-\left(\operatorname{Re}\left(\lambda_{k}\right)-\operatorname{Re}\left(\lambda_{j}\right)\right)} .
\end{aligned}
$$

Under condition (ii), the sum of the second factor is bounded. 
Theorem 3.3. Assume that the sequence $\Lambda=\left\{\lambda_{k}\right\}_{k=0}^{\infty}$ satisfies conditions (i) and (ii) in the previous lemma. Then $Z(s, \Lambda)$ is defined for $\operatorname{Re}(s)>1-\operatorname{Re}\left(\lambda_{0}\right)$. Moreover $Z(s, \Lambda)$ has an analytic continuation as a meromorphic function on $s \in \mathbb{C}$.

Proof. We see from the lemma that

$$
\left|\sum_{k=0}^{\infty} \sum_{p} p^{-\left(s+\lambda_{k}\right)}\right| \ll \sum_{p} p^{-\operatorname{Re}(s)-\operatorname{Re}\left(\lambda_{0}\right)} .
$$

The right side converges in $\operatorname{Re}(s)>1-\operatorname{Re}\left(\lambda_{0}\right)$. Hence $Z(s, \Lambda)$ is holomorphic on this domain. Further, we have

$$
Z\left(s,\left\{\lambda_{k}\right\}_{k=0}^{\infty}\right)=\prod_{k=0}^{j-1} \zeta\left(s+\lambda_{k}\right) \cdot \prod_{k=j}^{\infty} \zeta\left(s+\lambda_{k}\right)=\prod_{k=0}^{j-1} \zeta\left(s+\lambda_{k}\right) \times Z\left(s,\left\{\lambda_{k}\right\}_{k=j}^{\infty}\right)
$$

for any $j$. From the previous lemma, we see that the second factor is now defined for $\operatorname{Re}(s)>1-\operatorname{Re}\left(\lambda_{j}\right)$. This provides a meromorphic continuation to $\mathbb{C}$.

We remark that the higher Riemann zeta function has the Dirichlet series expression $Z(s, \Lambda)=\sum_{n=1}^{\infty} g_{\Lambda}(n) n^{-s}$, where

$$
g_{\Lambda}(n)=\sum_{\substack{n_{0} \cdot n_{1} \cdot n_{2} \ldots \ldots=n \\ n_{0} \geq 1, n_{1} \geq 1, \cdots}} n_{0}^{-\lambda_{0}} \cdot n_{1}^{-\lambda_{1}} \cdot n_{2}^{-\lambda_{2}} \cdots
$$

From this expression, we see that $g_{\Lambda}(n)$ is multiplicative and $Z(s, \Lambda)$ has the Euler product

$$
Z(s, \Lambda)=\prod_{p: \text { prime }} \sum_{m=0}^{\infty} \frac{g_{\Lambda}\left(p^{m}\right)}{p^{m s}}, \quad \text { for } \operatorname{Re}(s)>1-\operatorname{Re}\left(\lambda_{0}\right) .
$$

Moreover, using the Tauberian theorem (cf. $[\mathrm{Mu}]$ ), we obtain the behavior of $g_{\Lambda}(n)$ as follows.

Corollary 3.4. If $\Lambda=\left\{\lambda_{k}\right\}_{k=0}^{\infty}$ satisfies (i) and (ii), and $0 \leq \lambda_{0}=\cdots=\lambda_{K-1}<$ $\lambda_{K} \leq \cdots$, then we have

$$
\sum_{n \leq x} g_{\Lambda}(n)=\left(c_{\Lambda}+o(1)\right) x^{1-\lambda_{0}} \cdot \log ^{K-1} x \quad \text { as } x \rightarrow \infty,
$$

where $c_{\Lambda}$ is a constant which is given by

$$
c_{\Lambda}:=\frac{1}{(K-1) !} \lim _{s \rightarrow 1-\lambda_{0}}\left(s-1+\lambda_{0}\right)^{K} Z(s, \Lambda)=\frac{1}{(K-1) !} Z\left(1-\lambda_{0},\left\{\lambda_{k}\right\}_{k=K}^{\infty}\right) .
$$

3.2. Regularized product expression. We first recall the regularized product expression of the Riemann zeta function.

Lemma 3.5 ([区] $)$. For $\operatorname{Re}(z)>1$ and $\operatorname{Re}(s)>1$, we have

$$
\begin{aligned}
\sum_{\rho \in R}(z-\rho)^{-s}= & z^{-s}+(z-1)^{-s}-\sum_{n=0}^{\infty}(z+2 n)^{-s} \\
& -\frac{1}{\Gamma(s)} \sum_{k=0}^{\infty} \sum_{p: \text { prime }} \frac{\log p}{p^{n z}}\left(\log p^{n}\right)^{s-1},
\end{aligned}
$$

where $R$ denotes the set of the non-trivial zeros of the Riemann zeta function. 
This relation follows by applying Weil's explicit formula. For a proof, see [De. From the lemma we see that the function $\sum_{\rho \in R}(z-\rho)^{-s}$ has a meromorphic continuation to $s \in \mathbb{C}$ and the Riemann zeta function has the regularized product expression

$$
\prod_{\rho \in R}\left(\frac{s-\rho}{2 \pi}\right)=\frac{s}{2 \pi} \cdot \frac{s-1}{2 \pi} \cdot \prod_{n=0}^{\infty}\left(\frac{s+2 n}{2 \pi}\right)^{-1} \cdot \zeta(s) .
$$

Further, using properties of the Hurwitz zeta function and the gamma function, we have

$$
\prod_{\rho \in R}\left(\frac{s-\rho}{2 \pi}\right)=2^{-1 / 2}(2 \pi)^{-2} \cdot s(s-1) \cdot \pi^{-s / 2} \cdot \Gamma\left(\frac{s}{2}\right) \cdot \zeta(s) .
$$

We remark that this function is invariant under $s \leftrightarrow 1-s$.

Next we describe the regularized product expression of the higher Riemann zeta function as follows.

Theorem 3.6. Let $\Lambda$ be a regularizable sequence. Then the higher Riemann zeta function $Z(s, \Lambda)$ has the following regularized product expression:

$$
\begin{aligned}
\prod_{\lambda \in \Lambda, \rho \in R}\left(\frac{s+\lambda-\rho}{2 \pi}\right)= & \prod_{\lambda \in \Lambda}\left(\frac{s+\lambda}{2 \pi}\right) \cdot \prod_{\lambda \in \Lambda}\left(\frac{s-1+\lambda}{2 \pi}\right) \\
& \cdot \prod_{\lambda \in \Lambda, n \geq 0}\left(\frac{s+\lambda+2 n}{2 \pi}\right)^{-1} \cdot Z(s, \Lambda) .
\end{aligned}
$$

Proof. From (3.1), we see that

$$
\begin{aligned}
\sum_{\lambda \in \Lambda} \sum_{\rho \in R}(z+\lambda-\rho)^{-s} & =\sum_{\lambda \in \Lambda}(z+\lambda)^{-s}+\sum_{\lambda \in \Lambda}(z+\lambda-1)^{-s}-\sum_{\lambda \in \Lambda} \sum_{n=0}^{\infty}(z+\lambda+2 n)^{-s} \\
& -\frac{1}{\Gamma(s)} \sum_{\lambda \in \Lambda} \sum_{n=1}^{\infty} \sum_{p} \frac{\log p}{p^{n(z+\lambda)}}\left(\log p^{n}\right)^{s-1}
\end{aligned}
$$

for $\operatorname{Re}(z)>1$ and large $\operatorname{Re}(s)$. We observe that the right-hand side is now meromorphic for any $s \in \mathbb{C}$. Therefore the dotted product $\bigoplus_{\lambda, \rho}\{(z+\lambda-\rho) / 2 \pi\}$ exists. Further we obtain the equation (3.2) by using the relation

$\log Z(z, \Lambda)=\sum_{\lambda \in \Lambda} \sum_{p} \sum_{n=1}^{\infty} \frac{1}{n p^{n(z+\lambda)}}=\mathrm{CT}_{s=0} \frac{1}{s}\left\{\frac{(2 \pi)^{s}}{\Gamma(s)} \sum_{\lambda \in \Lambda} \sum_{n=1}^{\infty} \sum_{p} \frac{\log p}{p^{n(z+\lambda)}}\left(\log p^{n}\right)^{s-1}\right\}$.

This completes the proof.

3.3. Semi-lattice and functional equation. Let $\Omega:=\left\{n_{1} \omega_{1}+\cdots+n_{r} \omega_{r} \mid n_{j} \in\right.$ $\left.\mathbb{Z}_{\geq 0}\right\}$ with $\operatorname{Re}\left(\omega_{j}\right)>0$. Then it is seen that $\Omega$ is a regularizable sequence. Now, we consider the higher Riemann zeta function for the semi-lattice $\Omega$.

Definition 3.7. Define the higher Riemann zeta function of the weight $\boldsymbol{\omega}=$ $\left(\omega_{1} \cdots \omega_{r}\right)$ by

$$
Z(s, \boldsymbol{\omega}):=Z(s, \Omega)=\prod_{n_{1}, \cdots, n_{r}=0}^{\infty} \prod_{p: \text { prime }}\left(1-p^{-\left(s+n_{1} \omega_{1}+\cdots+n_{r} \omega_{r}\right)}\right)^{-1} .
$$


We see that this product absolutely for $\operatorname{Re}(s)>1$ and $Z(s, \boldsymbol{\omega})$ has an analytic continuation as a meromorphic function on $s \in \mathbb{C}$. Obviously, this is invariant under the arrangement of $\omega_{j}$ 's.

Proposition 3.8. The higher Riemann zeta function $Z(s, \omega)$ defined for $\operatorname{Re}(s)>1$ has a meromorphic continuation to the whole complex plane and satisfies

$$
\begin{aligned}
& Z\left(s,\left(\omega_{1}, \cdots, \omega_{r-1}, \omega_{r}\right)\right)=Z\left(s,\left(\omega_{1}, \cdots, \omega_{r-1}\right)\right) \cdot Z\left(s+\omega_{r},\left(\omega_{1}, \cdots, \omega_{r-1}, \omega_{r}\right)\right), \\
& Z(s, \boldsymbol{\omega}) \times \prod_{\substack{1 \leq k_{1}<\cdots<k_{j} \leq r \\
1 \leq j \leq r}} Z\left(s+\omega_{k_{1}}+\cdots+\omega_{k_{j}}, \boldsymbol{\omega}\right)^{(-1)^{j}}=\zeta(s) .
\end{aligned}
$$

Proof. We observe that

$$
\begin{aligned}
& Z\left(s,\left(\omega_{1}, \cdots, \omega_{r-1}, \omega_{r}\right)\right)=\prod_{n=0}^{\infty} Z\left(s+n \omega_{r},\left(\omega_{1}, \cdots, \omega_{r-1}\right)\right) \\
& \quad=Z\left(s,\left(\omega_{1}, \cdots, \omega_{r-1}\right)\right) \cdot \sum_{n=0}^{\infty} Z\left(s+n \omega_{r}+\omega_{r},\left(\omega_{1}, \cdots, \omega_{r-1}\right)\right) \\
& \quad=Z\left(s,\left(\omega_{1}, \cdots, \omega_{r-1}\right)\right) \cdot Z\left(s+\omega_{r},\left(\omega_{1}, \cdots, \omega_{r-1}, \omega_{r}\right)\right) .
\end{aligned}
$$

Thus the relation (3.3) holds. By using (3.3) repeatedly, we have

$$
\begin{aligned}
\zeta(s)= & Z\left(s,\left(\omega_{1}\right)\right) \cdot Z\left(s+\omega_{1},\left(\omega_{1}\right)\right)^{-1} \\
= & Z\left(s,\left(\omega_{1}, \omega_{2}\right)\right) Z\left(s+\omega_{2},\left(\omega_{1}, \omega_{2}\right)\right)^{-1} \\
& \cdot Z\left(s+\omega_{1},\left(\omega_{1}, \omega_{2}\right)\right)^{-1} Z\left(s+\omega_{1}+\omega_{2},\left(\omega_{1}, \omega_{2}\right)\right) \\
= & \cdots=Z(s, \boldsymbol{\omega}) \times \prod_{\substack{1 \leq k_{1}<\cdots<k_{j} \leq r \\
1 \leq j \leq r}} Z\left(s+\omega_{k_{1}}+\cdots+\omega_{k_{j}}, \boldsymbol{\omega}\right)^{(-1)^{j}} .
\end{aligned}
$$

This completes the proof.

Next we show the regularized product expression of $Z(s, \boldsymbol{\omega})$ as follows.

Theorem 3.9. We have

$$
\begin{aligned}
& \prod_{n_{1}, \cdots, n_{r} \geq 0, \rho \in R}\left(\frac{s+n_{1} \omega_{1}+\cdots+n_{r} \omega_{r}-\rho}{2 \pi}\right) \\
& =\exp \left\{\left(-\frac{B_{r}(s, \boldsymbol{\omega})}{r !}-\frac{B_{r}(s-1, \boldsymbol{\omega})}{r !}+\frac{B_{r+1}\left(s,\left(2, \omega_{1}, \cdots, \omega_{r}\right)\right)}{(r+1) !}\right) \log (2 \pi)\right\} \\
& \quad \times \Gamma(s, \boldsymbol{\omega})^{-1} \cdot \Gamma(s-1, \boldsymbol{\omega})^{-1} \cdot \Gamma\left(s,\left(2, \omega_{1}, \cdots, \omega_{r}\right)\right) \cdot Z(s, \boldsymbol{\omega}) .
\end{aligned}
$$

Here $B_{n}(s, \boldsymbol{\omega})$ is the multiple Bernoulli polynomial and $\Gamma(s, \boldsymbol{\omega})$ is the multiple gamma function.

Proof. We note that

$$
\begin{aligned}
& \prod_{n_{1}, \cdots, n_{r}=0}^{\infty}\left(\frac{z+n_{1} \omega_{1}+\cdots+n_{r} \omega_{r}}{2 \pi}\right) \\
& =(2 \pi)^{-\zeta(0, z, \omega)} \prod_{n_{1}, \cdots, n_{r}=0}^{\infty}\left(z+n_{1} \omega_{1}+\cdots+n_{r} \omega_{r}\right) .
\end{aligned}
$$

Then, the result follows from Theorem 3.6. 
From this theorem, we see that the function

$$
\begin{aligned}
\hat{Z}(s, \boldsymbol{\omega}): & =\exp \left\{\left(-\frac{B_{r}(s, \boldsymbol{\omega})}{r !}-\frac{B_{r}(s-1, \boldsymbol{\omega})}{r !}+\frac{B_{r+1}\left(s,\left(2, \omega_{1}, \cdots, \omega_{r}\right)\right)}{(r+1) !}\right) \log (2 \pi)\right\} \\
& \times \Gamma(s, \boldsymbol{\omega})^{-1} \cdot \Gamma(s-1, \boldsymbol{\omega})^{-1} \cdot \Gamma\left(s,\left(2, \omega_{1}, \cdots, \omega_{r}\right)\right) \cdot Z(s, \boldsymbol{\omega})
\end{aligned}
$$

is an entire function of order $r+1$ with zeros at $s=\rho-n_{1} \omega_{1}-\cdots-n_{r} \omega_{r}$. Further $\hat{Z}(s, \boldsymbol{\omega})$ satisfies the relation

$$
\hat{Z}\left(s,\left(\omega_{1}, \cdots, \omega_{r-1}, \omega_{r}\right)\right)=\hat{Z}\left(s,\left(\omega_{1}, \cdots, \omega_{r-1}\right)\right) \cdot \hat{Z}\left(s+\omega_{r},\left(\omega_{1}, \cdots, \omega_{r-1}, \omega_{r}\right)\right) .
$$

Finally, we give the functional equation of the higher Riemann zeta function $Z(s, \boldsymbol{\omega})$ as follows.

Theorem 3.10. Define the function $\Lambda(s, \boldsymbol{\omega})$ by

$$
\Lambda(s, \boldsymbol{\omega}):=\hat{Z}(s, \boldsymbol{\omega}) \cdot \hat{Z}\left(1+\omega_{1}+\cdots+\omega_{r}-s, \boldsymbol{\omega}\right)^{(-1)^{r+1}} .
$$

Then we have

$$
\Lambda\left(s,\left(\omega_{1}, \cdots, \omega_{r-1}, \omega_{r}\right)\right)=\Lambda\left(s,\left(\omega_{1}, \cdots, \omega_{r-1}\right)\right) \cdot \Lambda\left(s+\omega_{r},\left(\omega_{1}, \cdots, \omega_{r-1}, \omega_{r}\right)\right) .
$$

Moreover, $\Lambda(s, \boldsymbol{\omega})$ satisfies the functional equation

$$
\Lambda(s, \boldsymbol{\omega}) \times \prod_{\substack{1 \leq k_{1}<\cdots<k_{j} \leq r \\ 1 \leq j \leq r}} \Lambda\left(s+\omega_{k_{1}}+\cdots+\omega_{k_{j}}, \boldsymbol{\omega}\right)^{(-1)^{j}}=1 .
$$

Proof. We observe (3.3) and

$$
\begin{aligned}
& \hat{Z}\left(1+\omega_{1}+\cdots+\omega_{r-1}+\omega_{r}-s,\left(\omega_{1}, \cdots,+\omega_{r-1}, \omega_{r}\right)\right) \\
= & \frac{\hat{Z}\left(1+\omega_{1}+\cdots+\omega_{r-1}+\omega_{r}-\left(s+\omega_{r}\right),\left(\omega_{1}, \cdots,+\omega_{r-1}, \omega_{r}\right)\right)}{\hat{Z}\left(1+\omega_{1}+\cdots+\omega_{r-1}-s,\left(\omega_{1}, \cdots, \omega_{r-1}\right)\right)} .
\end{aligned}
$$

Then the equation (3.4) follows immediately.

Next, using (3.4) repeatedly, we have

$$
\Lambda(s, \boldsymbol{\omega}) \times \prod_{\substack{1 \leq k_{1}<\cdots<k_{j} \leq r \\ 1 \leq j \leq r}} \Lambda\left(s+\omega_{k_{1}}+\cdots+\omega_{k_{j}}, \boldsymbol{\omega}\right)^{(-1)^{j}}=\hat{\zeta}(s) \cdot \hat{\zeta}(1-s)^{-1}=1,
$$

where

$$
\hat{\zeta}(s):=\prod_{\rho \in R}\left(\frac{s-\rho}{2 \pi}\right)=2^{-1 / 2}(2 \pi)^{-2} \cdot s(s-1) \cdot \pi^{-s / 2} \cdot \Gamma\left(\frac{s}{2}\right) \cdot \zeta(s) .
$$

Hence the theorem follows.

\section{REFERENCES}

[Ba] E.W.Barnes, On the theory of the multiple gamma function. Trans. Cambridge Philos. Soc. 19 (1904), 374-425.

[CL] H.Cohen, H.W.Lenstra, Heuristics on class groups of number fields. Lecture Notes in Math. 1068, Springer, Berlin (1984), 33-62. MR0756082 (85j:11144)

[De] C.Deninger, Local L-factors of motives and regularized determinants. Invent. Math. 107 (1992), 135-150. MR1135468 (93a:11056)

[Go] Y.Gon, Gamma factors of Selberg zeta functions and functional equation of Ruelle zeta functions. Math. Ann. 308 (1997), 251-278. MR1464902 (98h:11109)

[HKW] M.Hirano, N.Kurokawa, M.Wakayama, Half zeta functions. J. Ramanujan Math. Soc. 18 (2003), 195-209. MR1995867 (2004m:11136) 
[HW] Y.Hashimoto, M.Wakayama, Hierarchy of the Selberg zeta functions. Preprint (2004).

[IIl] G.Illies, Regularized products and determinants. Comm. Math. Phys. 220 no.1 (2001), 69-94. MR1882400 (2003f:11131)

[JL] J.Jorgenson, S.Lang, Basic analysis of regularized series and products. Lecture Notes in Math. 1564, Springer, Berlin (1993). MR.1284924 (95e:11094)

[KK] N.Kurokawa, S.Koyama, Multiple sine functions. Forum Math. 15 (2003), 839-876. MR2010282 (2004g:11077)

[Ku1] N.Kurokawa, Lectures on multiple sine functions. Notes by Shinya Koyama, Univ. of Tokyo (1991), April-July.

[Ku2] N.Kurokawa, Gamma factors and Plancherel measures. Proc. Japan Acad. 68 Ser. A (1992), 256-260. MR 1202627 (94b:11044)

[KMW] N.Kurokawa, S.Matsuda, M.Wakayama, Gamma factors and functional equations of higher Riemann zeta functions. Preprint (2004).

[KW1] N.Kurokawa, M.Wakayama, Zeta extensions. Proc. Japan Acad. 78 Ser. A (2002), 126130. MR1930216 (2003h:11112)

[KW2] N.Kurokawa, M.Wakayama, Higher Selberg zeta functions. Comm. Math. Phys. 247 (2004), 447-466. MR2063268 (2005h:11200)

[KW3] N.Kurokawa, M.Wakayama, Generalized zeta regularizations, quantum class number formulas, and Appell's $O$ functions. to appear in The Ramanujan J.

[Mo] T.Momotani, Higher Selberg zeta functions for congruence subgroups. Preprint (2004).

$[\mathrm{Mu}]$ M.R.Murty, V.K.Murty, Non-vanishing of L-functions and applications. Progress in Mathematics 157 (1997). MR:1482805 (98h:11106)

[Pa] S.J.Patterson, An introduction to the theory of the Riemann zeta function. Cambridge Studies in Advanced Mathematics 14 (1988). MR0933558 (89d:11072)

[We] A.Weil, Sur les "formules explicites" de la théorie des nombers premiers. Comm. Sém. Math. Univ. Lund (1952), 252-265. MR0053152 (14:727e)

Graduate School of Mathematics, Kyushu University 6-10-1, Hakozaki Fukuoka 812 8581, JAPAN

E-mail address: momo@math.kyushu-u.ac.jp 